\title{
Quantitative analysis of two isoflavones in Pueraria lobata flowers from eleven Chinese provinces using high performance liquid chromatography
}

\author{
Meicun Yao', Yiting Liao', George Q Li², Francis CP Law³ and Yi Tang*1
}

\begin{abstract}
Background: Pueraria lobata flower (Gehua) is a medicinal herb to treat intoxication, hepatic and gastrointestinal tract lesion induced by alcohol. This study aims to develop a new HPLC method for the determination of two major isoflavones in P. lobata flowers, namely tectoridin and 6"-O-xylosyl-tectoridin.

Methods: A high performance liquid chromatography (HPLC) method with a $\mathrm{C}_{18}$ column ( $\left.250 \mathrm{~mm} \times 4.6 \mathrm{~mm}, 5 \mu \mathrm{m}\right)$ was developed for the quantitative analysis of tectoridin and 6"-O-xylosyl-tectoridin, the main isoflavone components in P. lobata flower. A simple gradient of acetonitrile/water (0 min 15:85; 35 min 50:50; 36 min 15:85; 40 min 15:85; v/v) was used, and $265 \mathrm{~nm}$ was selected as detection wavelength. Tectoridin and 6"-O-xylosyl-tectoridin were used as the external standards in quality control of P. lobata flower for the first time. The method was applied to practical use in quality assessment of eleven batches of $P$. lobata flower samples in Chinese herbal medicine market.
\end{abstract}

Results: The peak area response was linear for tectoridin in the $11.8-236.4 \mu \mathrm{g} / \mathrm{mL}$ range with a correlation coefficient of 0.9996 ( $P<0.001$ ), and for 6"-O-xylosyl-tectoridin in the $10.33-185.99 \mu \mathrm{g} / \mathrm{mL}$ range with a correlation coefficient of $0.9984(\mathrm{P}<0.001)$ respectively. The average recoveries were $102.7-103.7 \%$ for tectoridin and $95.7-103.2 \%$ for 6 "-Oxylosyl-tectoridin (RSDs $<3 \%$ ), and the intra-day and inter-day RSDs of the two components were less than $2 \%$. This HPLC method was applied to assess the quality of P. lobata flower from eleven provinces in China. P. lobata flowers from northern China contained 26.46-43.28 mg/g of tectoridin and 30.90-48.23 mg/g of 6"-O-xylosyl-tectoridin comparing to $10.00-19.81 \mathrm{mg} / \mathrm{g}$ of tectoridin and $11.08-37.03 \mathrm{mg} / \mathrm{g}$ of 6"-O-xylosyl-tectoridin in those from southern China.

Conclusion: The results showed that P. lobata flowers from northern China contained more tectoridin and 6"-Oxylosyl-tectoridin than those from southern China.

\section{Background}

Pueraria lobata (Willd) Ohwi is a common Chinese medicinal plant that belongs to the Leguminosae family. While the P. lobata root (Gegen) is beneficial for cardiovascular diseases, the P. lobata flower (Gehua) is used to treat intoxication, hepatic and gastrointestinal (GI) tract lesion induced by alcohol [1]. P. lobata flower reduces ethanol absorption by the GI tract [2,3] and modulates the immune and endocrine systems to alleviate the damage caused by alcohol to the hepatic and GI functions. $P$. lobata flower has anti-diabetic [4], anti-stress [5], antiviral [6] and antioxidant [7] properties and induces apop-

* Correspondence: tigertang@21cn.com

1 School of Pharmaceutical Sciences, Sun Yat-sen University, Guangzhou 510006, Guangdong, PR China

Full list of author information is available at the end of the article tosis in human neuroblastoma cells [8]. It is also used to treat rectal ulcer and bleeding [9].

Most of pharmacological effects of $P$. lobata flower have been attributed to its isoflavone components [2,3,5,6,8-10], e.g. kakkalide, kakkalidone, puerarin, irisolidone, 6"-O-xylosyl-glycitin, tectoridin and 6"-Oxylosyl-tectoridin (Figure 1); thus, determination of isoflavone is crucial for the quality control of $P$. lobata flower [11].

Chemical components in P. lobata flowers undergo changes under storage conditions. Tectoridin is the major isoflavone component during the first-5-year storage and nearly no kakkalide is detected [12]. In this study, we also found that tectoridin was the major component of ethanol $(70 \%)$ extract of $P$. lobata flower. Tectoridin is used as 


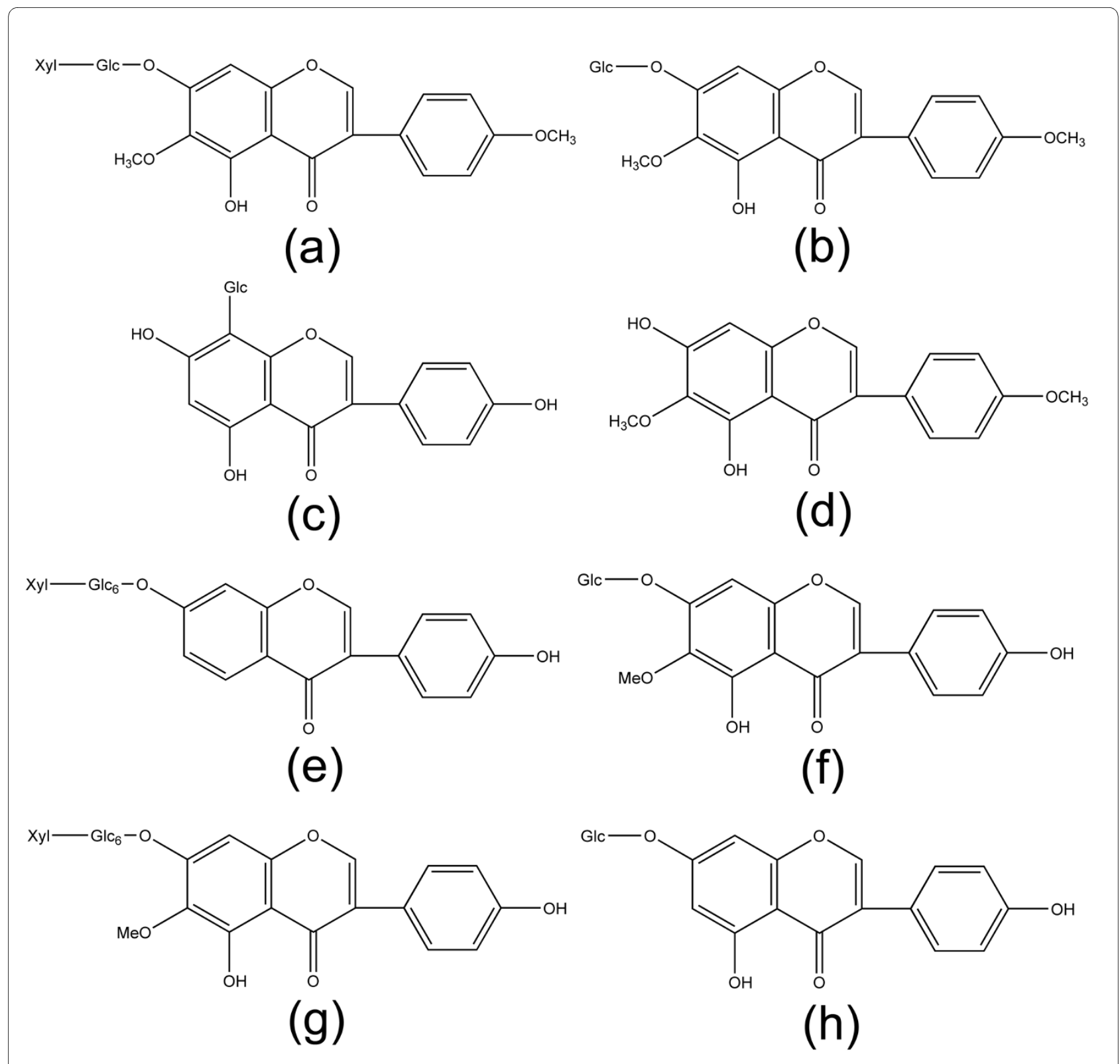

Figure 1 Chemical structures of isoflavones in P. lobata flower. (a) kakkalide, (b) kakkalidone, (c) puerarin, (d) irisolidone, (e) 6"-O-xylosyl-glycitin, (f) tectoridin, (g) 6"-O-xylosyl-tectoridin, (h) genistin.

a marker compound for the quality evaluation of Belamcanda chinensis in the Chinese Pharmacopoeia [13]. While tectoridin is not a characteristic component of $P$. lobata flower, it may be used as the marker compound for $P$. lobata flowers. Tectorigenin, the major metabolite of tectoridin, showed potent pharmacological effects on ethanol-induced diseases $[2,5,8]$.

Several methods with isoflavone determination for $P$. lobata flower have been reported [12,14-16]. For example, an ultraviolet (UV) spectrophotometry method to determine the isoflavone content with kakkalide as the marker was developed [16]. However, puerarin is often used instead of kakkalide in China where kakkalide is not widely available. The UV method is not satisfactory as the maximum absorbance wavelength of puerarin $(250 \mathrm{~nm})$ is different from that of kakkalide $(270 \mathrm{~nm})$. A high performance liquid chromatography (HPLC) analysis with chloroform, methanol and distilled water was used to determine the freshness of $P$. lobata flower [12]. Another HPLC method with isoflavones genistin and genistein as 
external standards was developed for quality control of the three isoflavones in P. lobata flower, namely 6"-Oxylosyltectoridin, tectoridin and tectorigenin [15].

A new HPLC analytical method was developed in this study, which was with a reversed phase column and a UV detector for the quantitative determination of the two major isoflavones in $P$. lobata flower, namely tectoridin and 6"-O-xylosyl-tectoridin. The same kinds of isoflavone standards were used as the external standards, whereby two major isoflavones from eleven batches of $P$. lobata flowers were determined.

\section{Methods}

\section{Materials and chemicals}

P. lobata flowers from eleven provinces in China were purchased from Guangzhou Medicinal Materials Co. (Guangzhou, China). The samples were from eleven locations of production, namely Hebei, Shanxi, Shandong, Henan, Jiangsu, Anhui and Hubei (provinces in northern China) as well as Jiangxi, Hunan, Guangxi and Guangdong (provinces in southern China). The authenticity of production were certified by Jun Wang, Associate Professor, School of Pharmaceutical Sciences, Sun Yat-sen University (Guangzhou, China), by observation of the shapes and microscopic characteristics, and properties tests according to Guangdong Chinese Materia Medica Standards [17]. Voucher specimens were deposited in the School of Pharmaceutical Sciences, Sun Yat-sen University (Guangzhou, China).

Reference standards of tectoridin and 6"-O-xylosyl-tectoridin were purchased from the National Institute for the Control of Pharmaceutical and Biological Products of China (Beijing, China). The purity for all reference standards was over $99 \%$. HPLC grade acetonitrile, methanol and analytical grade ethanol for isoflavones extraction were obtained from Hanbang Chemical Reagent Co. (China). HPLC-grade water was obtained from Millipore Elix 3 Progard 2 Water System (Millipore, USA).

\section{Preparation of standard solutions}

Stock solution of tectoridin $(236.4 \mu \mathrm{g} / \mathrm{mL})$ was prepared by dissolving $11.82 \mathrm{mg}$ of tectoridin in $50 \mathrm{~mL}$ of $80 \%(\mathrm{v} / \mathrm{v})$ methanol solution. Stock solution of 6"-O-xylosyl-tectoridin $(186.0 \mu \mathrm{g} / \mathrm{mL})$ was prepared by dissolving $9.3 \mathrm{mg}$ of $6 "$-O-xylosyl-tectoridin in $50 \mathrm{~mL}$ of $80 \%(\mathrm{v} / \mathrm{v})$ methanol solution. The stock solutions were stored at $4{ }^{\circ} \mathrm{C}$ and used within one month. Working solutions were prepared by serial dilutions of the stock solution; 47.3, 94.6, $189.1 \mu \mathrm{g} /$ $\mathrm{mL}$ of tectoridin and 37.2, 148.8, $186.0 \mu \mathrm{g} / \mathrm{mL}$ of $6 "-\mathrm{O}-$ xylosyl-tectoridin were used as the quality control solutions. The standard curve was plotted according to the linear least-squares using the peak areas (Excel 2000, Microsoft, USA).

\section{Sample preparation}

Dry samples were pulverized to fine powder, $8 \mathrm{~g}$ of which was accurately weighed and immersed in $80 \mathrm{~mL}$ of $70 \%$ $(\mathrm{v} / \mathrm{v})$ ethanol solution for 60 minutes. It was then refluxed in a water bath $\left(88^{\circ} \mathrm{C}\right)$ for 60 minutes and filtered; the residue was further refluxed twice in $64 \mathrm{~mL}$ of $70 \%(\mathrm{v} / \mathrm{v})$ ethanol solution for 60 minutes under the same condition. Combined filtrates were evaporated to dryness and further vacuum dried in an oven. About $20 \mathrm{mg}$ of the powder was dissolved in $50 \mathrm{~mL}$ of $80 \%(\mathrm{v} / \mathrm{v})$ methanol with sonication. Once cooled to room temperature, the sample solution was filtered through a $0.45 \mu \mathrm{m}$ syringe membrane filter (Millipore, USA). The filtrate $(10 \mu \mathrm{L})$ was injected into Waters 717-1525-2996 HPLC system (Waters, USA) for analysis.

\section{Statistical analysis}

Linearity analysis was performed according to linear least-squares regression with Excel 2000 (Microsoft, USA). Calculation of relative standard deviation (RSD) was also carried out with Excel 2000 (Microsoft, USA). One-way ANOVA $(\alpha=0.05)$ was applied in the means comparison of extraction yields and isoflavone determination results of samples from northern and southern provinces with SPSS 16.0 (IBM, USA). P $<0.05$ was considered statistically significant.

\section{Instruments and the chromatographic conditions}

The Waters HPLC system was equipped with a binary pump, auto-sampler and Waters 2487 dual wavelengths absorbance detector (Waters, USA). All separations were performed on a GRACE $\mathrm{C}_{18}$ column $(250 \mathrm{~mm} \times 4.6 \mathrm{~mm}$, $5 \mu \mathrm{m}$ ) (W.R. Grace, USA). Mobile phase was a gradient of acetonitrile/water (0 min $15: 85 ; 35 \mathrm{~min} 50: 50 ; 36 \mathrm{~min}$ $15: 85 ; 40 \mathrm{~min} 15: 85$; v/v). Flow rate was $0.8 \mathrm{~mL} / \mathrm{min}$. Quantification was performed with a UV detector (Waters, USA) at $265 \mathrm{~nm}$. Injection volume was $10 \mu \mathrm{L}$. There was a 10-minute interval between sample injections. The column was kept at ambient temperature.

\section{Calibration}

Obtained with seven concentrations in triplicates, the calibration curve was plotted according to the linear regression analysis of the integrated peak areas $(y$-axis) versus concentrations $(x$-axis, $\mu \mathrm{g} / \mathrm{ml})$ of tectoridin and 6"-O-xylosyl-tectoridin. Limit of detection (LOD) and limit of quantification (LOQ) were determined with standard solution at a signal-to-noise ratio of three and ten respectively.

\section{Precision}

We studied the intra-day and inter-day variability to evaluate the precision of the method. Three solutions (high, medium and low concentrations) containing 30.5, 60.9, $118.5 \mu \mathrm{g} / \mathrm{mL}$ of tectoridin and $38.1,69.4,129.7 \mu \mathrm{g} / \mathrm{mL}$ of 
6"-O-xylosyl-tectoridin were prepared. The quantity of each component was determined by the respective calibration curve. RSD was used to measure precision. The inter-day reproducibility test was carried out on three different days.

\section{Repeatability}

Six independently prepared extracts of $P$. lobata flower from Henan province, China were analyzed and the repeatability was assessed by calculating the RSDs in six measurements.

\section{Recovery}

Recovery studies were carried out by spiking three concentrations of mixed standards of tectoridin and 6"-Oxylosyl-tectoridin to the P. lobata flower sample from Henan. The spiked samples were extracted and analyzed. Recovery $(\%)=\left(\mathrm{A}_{\mathrm{s}}-\mathrm{A}\right) / \mathrm{A}_{\mathrm{a}}{ }^{*} 100 \%$. $\mathrm{A}_{\mathrm{s}}$ refers to the amount of tectoridin or 6"-O-xylosyl-tectoridin found after spiking of the mixed standards whereas $A$ refers to the amount of those found before spiking. And $A_{a}$ refers to the amount of reference standards actually added to the sample. The average recoveries of every spiking concentration and the RSDs of recoveries in each concentration groups were calculated.

\section{Results}

\section{Optimization of the chromatographic conditions}

Ultraviolet scanning from wavelength $200 \mathrm{~nm}$ to $400 \mathrm{~nm}$ was carried out for P. lobata flower $70 \%$ ethanol extract and tectoridin standard $80 \%(\mathrm{v} / \mathrm{v})$ methanol solution. Both showed maximum absorbance at $265 \mathrm{~nm}$, which was different from those of kakkalide $(270 \mathrm{~nm})$ and puerarin $(250 \mathrm{~nm})$, indicating that tectoridin was more representative for total isoflavone of $P$. lobata flower than puerarin.

The composition of the mobile phase was optimized by comparing solvents and gradient profiles. The methanol mobile phase showed a baseline drift whereas acetonitrile mobile phase produced better separation and shorter run time. Retention time $\left(t_{\mathrm{R}}\right)$ of tectoridin and 6"-O-xylosyltectoridin were $22.4 \mathrm{~min}$ and $18.2 \mathrm{~min}$ with methanol/ water as mobile phase, whereas $t_{R}$ of the two isoflavone were $17.7 \mathrm{~min}$ and $15.6 \mathrm{~min}$ respectively with acetonitrile/water. Samples were eluted with a linear gradient from $15 \%$ to $50 \%$ acetonitrile/water over $35 \mathrm{~min}$, then from $50 \%$ back to $15 \%$ over $36 \mathrm{~min}$. The column was then washed for $4 \mathrm{~min}$ with this mobile phase ratio to the original conditions. Each HPLC run was within 40 minutes.

Resolutions between tectoridin, 6"-O-xylosyl-tectoridin and other peaks were larger than 1.5 (Figure 2).

\section{Calibration, LOD and LOQ}

The integrated chromatographic peak areas were plotted against the corresponding concentration of the standard solutions to obtain the calibration curves. The peak area response was linear for tectoridin in the 11.8-236.4 $\mu \mathrm{g} /$ $\mathrm{mL}$ range with a correlation coefficient of $0.9996(\mathrm{P}<$ 0.001 ), and for 6"-O-xylosyl-tectoridin in the 10.33$185.99 \mu \mathrm{g} / \mathrm{mL}$ range with a correlation coefficient of $0.9984(\mathrm{P}<0.001)$ respectively. The regression equations for the linear portion of the standard curve of tectoridin and 6"-O-xylosyl-tectoridin were $y=34920 x-1156.5$ and $y$ $=30731 x-216635$ respectively $(y$ refers to the peak area, $x$ concentration of the reference).

The LOD and LOQ for tectoridin were $24 \mathrm{ng} / \mathrm{mL}$ and $60 \mathrm{ng} / \mathrm{mL}, 80$ and $332 \mathrm{ng} / \mathrm{mL}$ for 6"-O-xylosyl-tectoridin respectively.

The results indicated a linear correlation between the peak area and concentration. Moreover, the method was sufficiently sensitive for the determination.

\section{Precision}

The instrument precision was assessed with intra-day and inter-day analyses using six injections of the standard solutions in duplicates. The intra-day and inter-day RSDs of the two components were less than $2 \%$ (Table 1 ).

\section{Repeatability}

RSDs of tectoridin and 6"-O-xylosyl-tectoridin of six independently prepared extracts of $P$. lobata flower from Henan were $0.90 \%$ and $0.78 \%$ respectively, indicating that the conditions used in the quantitative analysis were satisfactory.

\section{Recovery}

Aliquots of the two isoflavone standards were added to $P$. lobata flower from Henan. The mixtures were then extracted and analyzed with the described HPLC method. The average recoveries were $102.7-103.7 \%$ for tectoridin and 95.7-103.2\% for 6"-O-xylosyl-tectoridin (RSDs $<3 \%$ ), indicating that the method was sufficiently accurate for the determination.

\section{Sample analysis}

Means comparison of extraction yields and isoflavone determination results was performed according to oneway ANOVA. No significant difference in extraction yield was found among samples from the eleven provinces $(\mathrm{P}=$ 0.096), and extracts from northern China contained more isoflavones than those from southern China $(\mathrm{P}<0.001$ for tectoridin and $\mathrm{P}=0.023$ for $6 "$-O-xylosyl-tectoridin) whereas extracts from Guangdong had less tectoridin but more 6"-O-xylosyl-tectoridin comparing to those from northern China (Table 2). 


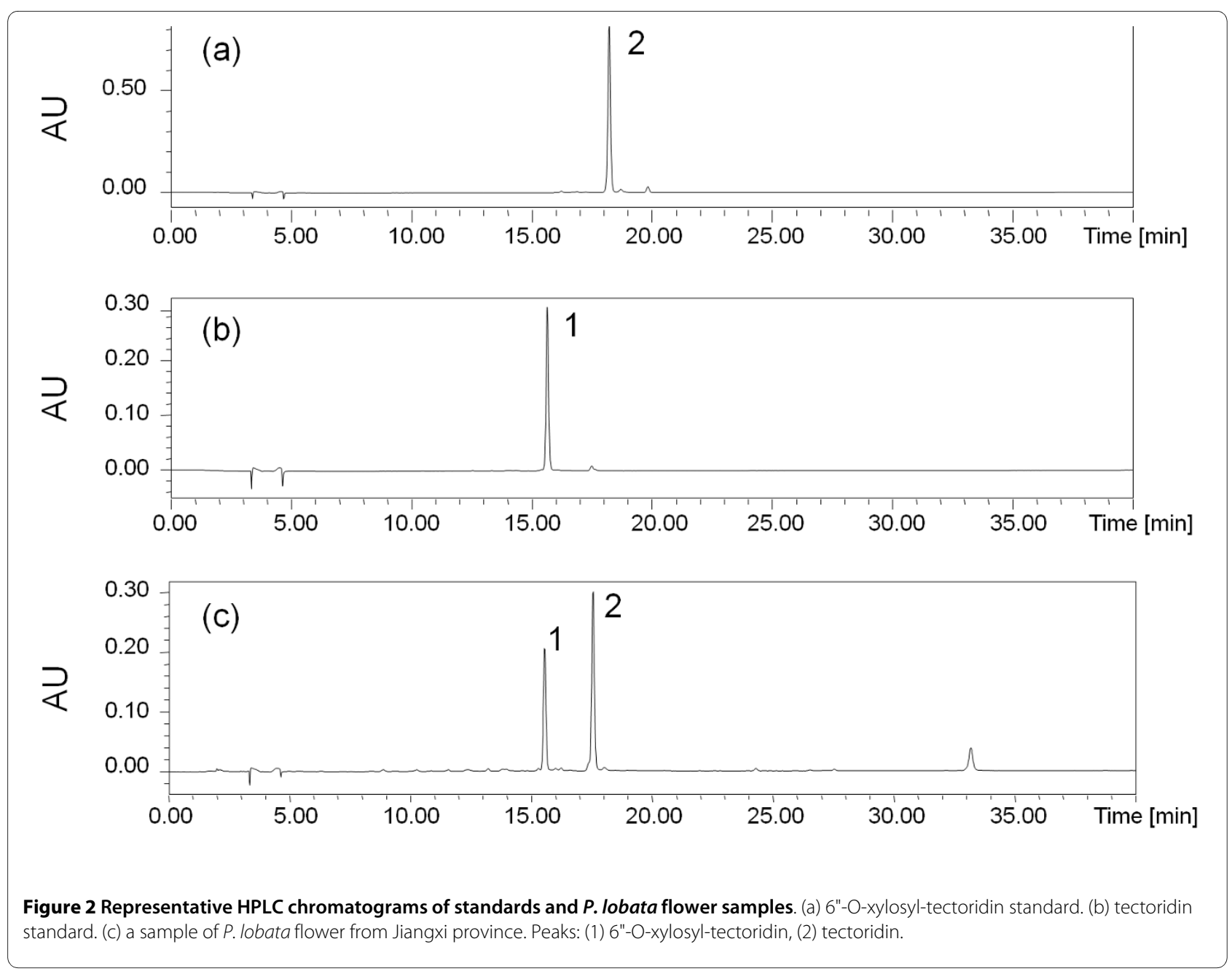

\section{Discussion}

The HPLC method developed in this study demonstrated better separation of isoflavones and better peak shapes than those offered in previous studies [12]. Moreover, the detection wavelength described in a previous study was $262 \mathrm{~nm}$ [15] whereas this study found that $265 \mathrm{~nm}$ was the maximum absorbance wavelength for both $P$. lobata flower extract and tectoridin standard solution. Therefore, tectoridin was more representative for total isoflavones of $P$. lobata flower and $265 \mathrm{~nm}$ as the detection wavelength was better than $262 \mathrm{~nm}$.

In Chinese medicine, medicinal herbs with high quality and efficacy from certain geographical locations are referred to as Daodi ('genuine'). For example, Frifllaria

Table 1: Precision of determination for the standards of two major isoflavones

\begin{tabular}{llll}
\hline Compounds & $\begin{array}{l}\text { Concentration } \\
(\boldsymbol{\mu} \mathbf{g} / \mathbf{m L})\end{array}$ & $\begin{array}{c}\text { Intra-day RSD\% } \\
(\boldsymbol{n}=\mathbf{6})\end{array}$ & $\begin{array}{c}\text { Inter-day RSD\% } \\
(\boldsymbol{n}=\mathbf{6})\end{array}$ \\
\hline \multirow{2}{*}{ Tectoridin } & 30.5 & 1.90 & 1.97 \\
& 60.9 & 1.03 & 1.86 \\
& 118.5 & 0.82 & 1.52 \\
6"-O-xylosyl-tectoridin & 38.1 & 1.65 & 1.98 \\
& 69.4 & 0.78 & 1.95 \\
\hline
\end{tabular}


Table 2: Determination of tectoridin and 6"-O-xylosyl-tectoridin in P. lobata flowers from eleven Chinese provinces

\begin{tabular}{llll}
\hline Province & Extraction yield (\%) & Tectoridin $(\mathbf{m g} / \mathbf{g}, \boldsymbol{n}=\mathbf{3})$ & $\mathbf{6}$--0-xylosyl-tectoridin $(\mathbf{m g} \mathbf{g}, \boldsymbol{n}=\mathbf{3})$ \\
\hline Hebei & 31.04 & $40.35(0.88)$ & $39.03(0.77)$ \\
Shanxi & 31.10 & $38.26(0.90)$ & $41.89(0.74)$ \\
Shandong & 33.78 & $26.46(0.78)$ & $30.90(0.72)$ \\
Henan & 32.02 & $43.28(0.79)$ & $38.43(0.82)$ \\
Jiangsu & 31.16 & $36.92(0.83)$ & $32.69(0.43)$ \\
Anhui & 33.32 & $30.50(0.69)$ & $30.63(0.63)$ \\
Hubei & 34.85 & $30.48(0.60)$ & $48.23(0.95)$ \\
Jiangxi & 34.24 & $10.00(0.14)$ & $18.61(0.26)$ \\
Hunan & 31.70 & $19.81(0.44)$ & $25.91(0.31)$ \\
Guangxi & 32.80 & $14.47(0.26)$ & $11.08(0.25)$ \\
Guangdong & 32.85 & $14.85(0.28)$ & $37.03(1.05)$ \\
\hline
\end{tabular}

Extraction yield is the ratio of the amount of dry extract actually obtained, to the amount of dry herb samples used for extraction.

Data are expressed as mean (SD).

SD: standard deviation

Means comparison was performed according to one-way ANOVA with $a=0.05$.

$\mathrm{P}>0.05$ for comparing extraction yields.

$\mathrm{P}<0.05$ for comparing two major isoflavones in samples from northern and southern provinces.

thuubergii Miq. (Zhebeimu), a cough medicine, is only considered Daodi if it is from Zhejiang province, China [1]. However, no such preferential origin is currently available for $P$. lobata flowers. The present study suggests that $P$. lobata flowers from northern China may be preferred to those from southern China.

Large-scale studies with more samples will have to be carried out to confirm this variation in isoflavones of $P$. lobata flower, which is likely to have an impact on the improvement in quality and efficacy of the medicines prepared from this herb.

\section{Conclusion}

The present study develops a new HPLC method for the determination of the two main isoflavones in P. lobata flowers, namely tectoridin and 6"-O-xylosyl-tectoridin. The results showed that $P$. lobata flowers from northern China contained more tectoridin and 6"-O-xylosyl-tectoridin than those from southern China.

\section{Competing interests}

The authors declare that they have no competing interests.

\section{Authors' contributions}

MY and YT conceived, designed and coordinated the study. MY developed the HPLC method. YL collected the materials, helped develop the HPLC method, analyzed the samples and drafted the manuscript. GQL and FCPL helped design and improved the study, drafted the manuscript and gave many important advices on research methodology. All authors read and approved the final version of the manuscript.

\section{Acknowledgements}

This work was supported by the 2008 Fund of Open Laboratory at the Sun Yatsen University (Guangzhou, China) and was partially supported by the National
Institute of Complementary Medicine (Sydney, Australia). The authors would like to thank Jun Wang, School of Pharmaceutical Sciences, Sun Yat-sen University (Guangzhou, China) for his assistance in authenticating the P. lobata flowers and confirming their origins.

\section{Author Details}

1School of Pharmaceutical Sciences, Sun Yat-sen University, Guangzhou 510006, Guangdong, PR China, 2Herbal Medicines Research and Education Centre, Faculty of Pharmacy, University of Sydney, NSW 2006, Australia and 3 Department of Biological Sciences, Simon Fraser University, Burnaby, BC V5A 156, Canada

Received: 16 December 2009 Accepted: 23 April 2010

Published: 23 April 2010

\section{References}

1. Chinese Herbal Medicine Editorial Board of State Administration of Traditional Chinese Medicine: Chinese Herbal Medicine. Shanghai 1999.

2. Lee HW, Choo MK, Bae EA, Kim DH: $\beta$-Glucuronidase inhibitor tectorigenin isolated from the flower of Pueraria thunbergiana protects carbon tetrachloride induced liver injury. Liver Int 2003, 23(4):221-226.

3. Han YO, Han MJ, Park SH, Kim DH: Protective effects of kakkalide from Flos Puerariae on ethanol-induced lethality and hepatic injury are dependent on its biotransformation by human intestinal microflora. J Pharmacol Sci 2003, 93(3):331-336.

4. Choi J, Shin MH, Park KY, Lee KT, Jung HJ, Lee MS, Park HJ: Effect of kaikasaponin III obtained from Pueraria thunbergiana flowers on serum and hepatic lipid peroxides and tissue factor activity in the streptozotocin-induced diabetic rat. J Med Food 2004, 7:31-37.

5. Kang KA, Lee KH, Chae S, Zhang R, Jung MS, Kim SY, Kim HS, Kim DH, Hyun $\mathrm{JW}$ : Cytoprotective effect of tectorigenin, a metabolite formed by transformation of tectoridin by intestinal microflora, on oxidative stress induced by hydrogen peroxide. Eur J Pharmacol 2005, 519(12):16-23.

6. Kim SY, Kim DH, Hyun JW, Henson JW, Kim HS: Irisolidone, an isoflavone metabolite, represses JC virus gene expression via inhibition of Sp1 binding in human glial cells. Biochem Biophys Res Commun 2006, 344(1):3-8. 
7. Lee MK, Cho SY, Jang JY, Cho MS, Jeon SM, Jang MK, Kim MJ, Park YB: Effects of Puerariae Flos and Puerariae Radix extracts on antioxidant enzymes in ethanol-treated rats. Am J Chin Med 2001, 29(2):343-354.

8. Lee KT, Sohn IC, Kim YK, Choi JH, Choi JW, Park HJ, Itoh Y, Miyamoto K. Tectorigenin, an isoflavone of Pueraria thunbergiana Benth., induces differentiation and apoptosis in human promyelocytic leukemia $\mathrm{HL}-60$ cells. Biol Pharm Bull 2001, 24:1117-1121.

9. Kim CM, Shin MK, Ahn DK, Lee KS: Encyclopaedia of Chinese Medicine Seoul: Jungdam Publishers; 1998

10. Shin JE, Bae EA, Lee YC: Estrogenic effect of main components kakkalide and tectoridin of Puerariae Flos and their metabolites. Biol Pharm Bull 2006, 29:1202-1206

11. Park HJ, Park JH, Moon JO, Lee KT, Jung WT, Oh SR, Lee HK: Isoflavone glycosides from the flowers of Pueraria thunbergiana. Phytochemistyr 1999, 51(1):147-151.

12. Kim C, Shin S, Ha H, Kim JM: Study of substance changes in flowers of Pueraria thunbergiana Benth. during storage. Arch Pharm Res 2003, 26:210-213.

13. Li J, Li WZM, Huang W, Cheung AWH, Bi CWC, Duan R, Guo AJY, Dong TTX, Tsim KWK: Quality evaluation of Rhizoma Belamcandae (Belamcanda chinensis (L.) DC.) by using high-performance liquid chromatography coupled with diode array detector and mass spectrometry. $J$ Chromatogr A 2009, 1216(11):2071-2078.

14. Kurihara T, Kikuchi M: Studies on the constituents of flowers V. on the components of flower of Pueraria thunbergiana Benth. (2). Isolation of a new isoflavone glycoside. Yakugaku Zasshi 1975, 95(11):1283-1285.

15. Bebrevska L, Bravo L, Vandervoort J, Pieters L, Vlietinck A, Apers S: Development and validation of an HPLC method for quality control of Pueraria lobata flower. Planta Med 2007, 73(15):1606-1613.

16. Zhang $\mathrm{S}, \mathrm{He} \mathrm{Y}$, Liu B: Determination of kakkalide in the flowers of Pueraria Lobata by UV. J Changzhi Univ 2006, 23:11-12

17. Guangdong Food and Drug Administration: Guangdong Chinese Materia Medica Standards. Guangzhou 2005.

\section{doi: 10.1186/1749-8546-5-14}

Cite this article as: Yao et al., Quantitative analysis of two isoflavones in Pueraria lobata flowers from eleven Chinese provinces using high performance liquid chromatography Chinese Medicine 2010, 5:14

\section{Submit your next manuscript to BioMed Central} and take full advantage of:

- Convenient online submission

- Thorough peer review

- No space constraints or color figure charges

- Immediate publication on acceptance

- Inclusion in PubMed, CAS, Scopus and Google Scholar

- Research which is freely available for redistribution

Submit your manuscript at www.biomedcentral.com/submit
Ciomed Central 